\title{
SOME COMMENTS ON GARSIA NUMBERS
}

\author{
KEVIN G. HARE AND MAYSUM PANJU
}

\begin{abstract}
A Garsia number is an algebraic integer of norm \pm 2 such that all of the roots of its minimal polynomial are strictly greater than 1 in absolute value. Little is known about the structure of the set of Garsia numbers. The only known limit point of positive real Garsia numbers was 1 (given, for example, by the set of Garsia numbers $2^{1 / n}$ ). Despite this, there was no known interval of $[1,2]$ where the set of positive real Garsia numbers was known to be discrete and finite. The main results of this paper are:

- An algorithm to find all (complex and real) Garsia numbers up to some fixed degree. This was performed up to degree 40 .

- An algorithm to find all positive real Garsia numbers in an interval $[c, d]$ with $c>\sqrt{2}$.

- There exist two isolated limit points of the positive real Garsia numbers greater than $\sqrt{2}$. These are $1.618 \cdots$ and $1.465 \cdots$, the roots of $z^{2}-z-1$ and $z^{3}-z^{2}-1$, respectively. There are no other limit points greater than $\sqrt{2}$.

- There exist infinitely many limit points of the positive real Garsia numbers, including $\lambda_{m, n}$, the positive real root of $z^{m}-z^{n}-1$, with $m>n$.
\end{abstract}

\section{INTRODUCTION AND BACKGROUND}

We begin with a

Definition 1.1. A number $\gamma$ is a complex Garsia number if it is an algebraic integer with $\operatorname{norm}(\gamma)= \pm 2$, and such that $\gamma$ and all of $\gamma$ 's conjugates have absolute value strictly greater than 1 .

In particular, the minimal polynomial of a Garsia number looks like

$$
z^{n}+a_{n-1} z^{n-1}+\cdots+a_{1} z \pm 2,
$$

Typically, we will be interested in positive real Garsia numbers, although many of the theorems and results concerning them can be extended to the complex Garsia numbers. The purpose of this paper is to investigate the structure of the set of positive real Garsia numbers.

Some details about the set of positive real Garsia numbers are already known. For example, 2 (as the root of $z-2$ ) is the largest positive real Garsia number, and 1 is a limit point of the Garsia numbers (take, for example, the sequence of Garsia

Received by the editor September 7, 2011 and, in revised form, October 13, 2011.

2010 Mathematics Subject Classification. Primary 11K16, 11Y40.

The first author's research was partially supported by NSERC.

The second author's research was supported by NSERC, the UW President's Research Award, UW Undergraduate Research Internship, and the department of Pure Mathematics at the University of Waterloo.

Computational support provided by CFI/OIT grant. 
numbers that are the roots of the polynomials $z^{n}-2$ as $n$ approaches infinity). However, not much else is known about this set of numbers.

These numbers were originally looked at by Garsia [10] and were the roots of what Garsia called "Type B" polynomials. Let $A(z)$ denote the distribution function of a random variable which takes \pm 1 with equal probability. Define $F(z, \beta)$ as the infinite convolution

$$
F(z, \beta)=A\left(\frac{z}{\beta}\right) * A\left(\frac{z}{\beta^{2}}\right) * A\left(\frac{z}{\beta^{3}}\right) * \ldots
$$

This is known as a Bernoulli convolution. It is known that

Theorem (Garsia 1962, [10]). If $\gamma$ is a real positive Garsia number, then $F(z, 1 /|\gamma|)$ is absolutely continuous and has derivative bounded by

$$
\frac{2}{\prod\left(\left|\gamma_{i}\right|-1\right)}
$$

where the product is taken over the conjugates of $\gamma$.

In contrast, the Pisot numbers give a remarkably different situation. In this case Theorem (Erdős 1939 [9]). If $q$ is a Pisot number, then $F(z, 1 /|q|)$ is purely singular.

It is known that this Bernoulli convolution is either absolutely continuous or purely singular 12. See also [15, 20, for more on Bernoulli convolutions.

Recall that a Pisot number is an algebraic integer greater than 1 such that all of its conjugates are strictly less than 1 in absolute value. The structure of the set of all Pisot numbers is well understood. The set is known to be closed [16, with a smallest value of $1.324 \cdots$, the real root of $z^{3}-z-1$ [19]. Amara [1] gave a complete description of the set of all limit points of the Pisot numbers. Boyd [3, 4] has given an algorithm that will find all Pisot numbers in an interval, where, in the case of limit points, the algorithm can detect the limit points and compensate for them.

The structure of Garsia numbers is far less understood, although some work has been done on special cases. The minimal polynomials of positive real Garsia numbers up to degree 5 were investigated by E. Rodemich, as discussed in Garsia [10, p. 420].

Brunotte [5] looked at positive real Garsia numbers to show that

Theorem (Brunotte 2009 [5]). The trinomial Garsia numbers of degree d are of the form

$$
\left\{z^{d}-2\right\} \cup\left\{z^{d}-z^{q}-2: 0<q<d, \frac{d}{\operatorname{gcd}(d, q)} \text { odd or } \frac{q}{\operatorname{gcd}(d, q)} \text { even }\right\} \cup P_{d}^{*}
$$

where

$$
P_{d}^{*}=\left\{z^{d}-2 z^{d / 3}-2\right\}
$$

if $d \equiv 0 \bmod 3$ and $P_{d}^{*}=\emptyset$ otherwise.

Brunotte also gave all real positive Garsia numbers of degree 3 and 4 as

Theorem (Brunotte 2009 [5]).

$P_{3}=\left\{z^{3}-2, z^{3}-z^{2}-2, z^{3}-z-2, z^{3}-2 z-2, z^{3}-z^{2}+z-2, z^{3}+z^{2}-z-2, z^{3}-2 z^{2}+2 z-2\right\}$ 
and

$$
\begin{array}{r}
P_{4}=\left\{z^{4}-2, z^{4}-z^{2}-z-2, z^{4}-z^{2}+z-2, z^{4}-2 z^{3}+2 z-2, z^{4}+2 z^{3}-2 z-2,\right. \\
\left.z^{4}-z^{3}+z-2, z^{4}+z^{3}-z-2\right\} .
\end{array}
$$

Recently [6], Brunotte extended his result to quadrinomial positive real Garsia numbers to give

Theorem (Brunotte (preprint) [6]). Let $n, k \in \mathbb{N}, n \geq 5,0<2 k<n$ and $b, c, d \in$ $\mathbb{Z} \backslash\{0\}$. Then the following statements hold:

- The polynomial

$$
z^{n}+b z^{n-k}+c z^{k}+d
$$

is the minimal polynomial for a positive real Garsia number if and only if $b c=-1$ and $d=-2$.

- Let $\gamma$ be a positive real Garsia number with minimal polynomial

$$
z^{n}-z^{n-k}+z^{k}-2 \text { or } z^{n}+z^{n-k}-z^{k}-2,
$$

and let $\gamma^{\prime}$ be any conjugate of $\gamma$. Then

$$
\gamma<2^{1 / \max (2, k)} \text { and }\left|\gamma^{\prime}\right|<\sqrt{2} \text {. }
$$

Moreover, if $n$ is odd, then all other conjugates of $\gamma$ are nonreal, and if $n$ is even, then $\gamma$ has exactly one other real conjugate, and this conjugate is negative.

In Table 5 we list all positive real Garsia numbers of degree at most 6 . The fact that all of these polynomials in this table have only one positive real root is a small number phenomenon, and is not true in general. See, for example, Table 1, A complete list of all Garsia numbers, including complex Garsia numbers, of degree at most 40 can be found at 11 .

Recall that a Garsia number has the property that its minimal polynomial $g(z)$ has $g(0)= \pm 2$. The case where $g(z)$ has a positive real root, and the minimal polynomial satisfies the stronger condition that $g(0)=2$, is also known as a canonical number system, or CNS polynomial. Kovács [13] has found all CNS polynomials up to degree 8 using exhaustive methods, along with some sophisticated bounds on the coefficients of CNS polynomials. This was later extended by Burcsi and Kovács [7] using a modified algorithm by Schur [17, 18] to find all CNS polynomials up to degree 11. We discuss and modify this method in Section 2, This new algorithm can go considerably further, and has been used up to degree 40 with no difficulties. This code can be found at 11. As well, a restricted search for positive real Garsia numbers in an interval $[c, d]$ with $c>\sqrt{2}$ can be performed.

Some observations from the restricted search are discussed in Section 3 . In particular, we show that

Theorem 1.1. There exist two isolated limit points of the positive real Garsia numbers greater than $\sqrt{2}$. These are $1.618 \cdots$ and $1.465 \cdots$, the roots of $z^{2}-z-1$ and $z^{3}-z^{2}-1$, respectively. There are no other limit points greater than $\sqrt{2}$.

A first easy result about Garsia numbers is

Lemma 1.1. If $g(z)$ is the minimal polynomial for a Garsia number $\gamma$, then $g\left(z^{n}\right)$ has the Garsia number $\gamma^{1 / n}$ as a root. 
Hence every Garsia number gives rise to an infinite family of Garsia numbers tending to 1 .

The existence of limit points other than 1, as shown in Theorem 1.1, is somewhat surprising. We can show a stronger result, namely that

Theorem 1.2. Let $\lambda_{n, m}$ be the positive real root of $z^{n}-z^{m}-1$ with $n>m>0$. Then $\lambda_{n, m}$ is a limit point of positive real Garsia numbers. In particular, for $n>m \geq 1$ and $k \geq 1$, we have that

$$
z^{k}\left(z^{n}-z^{m}-1\right) \pm\left(z^{n}-z^{m}+z^{n-m}-2\right)
$$

has a Garsia number as a root.

This result is discussed in Section 4 .

There is a real positive root in of equation (11), which tends to $\lambda_{n, m}$ as $k$ tends to infinity. We denote these roots as $\alpha_{n, m, k}$ and $\beta_{n, m, k}$ with $\alpha$ coming from "-" and $\beta$ coming from " + " in the choice for \pm . With the philosophy of Pisot numbers in mind, we call such Garsia numbers the regular Garsia numbers. It is not immediately clear if there is such a thing as an irregular Garsia number. The existence of irregular Garsia numbers is shown in Section [5. This section also discusses more on the structure of regular Garsia numbers. We further give an algorithm to determine if a positive real Garsia number is regular or irregular.

In Section 6, we explore computationally the possibility of other limit points. Computationally, it appears that the limit points $\lambda_{n, m}$ are in fact the only limit points, leading to the following:

Conjecture 1.1. Aside from the limit point 1, all limit points of the positive real Garsia numbers are roots of $z^{n}-z^{m}-1$ for some $n>m$.

Inspired by the result on Pisot numbers, we also make the following:

Conjecture 1.2. If $\gamma$ is a positive real Garsia number, sufficiently close to a root of $z^{n}-z^{m}-1$, then $\gamma$ is a polynomial in the form (5). Here, "sufficiently close" is dependent on $n$ and $m$.

Section 7 discusses an interesting construction of McKee and Smyth that gives rise to a large number of real positive Garsia numbers. Their observation is that if $p(z)$ and $q(z)$ are products of cyclotomic polynomials, such that $p(0)=q(0)$ and the roots of $p(z)$ and $q(z)$ are interlaced on the unit disc, then $g(z)=p(z)+q(z)$ is a (possibly complex) Garsia polynomial. Furthermore, they completely classify all such $p(z)$ and $q(z)$ with these properties. Given such a construction, it is easy to analyze the roots of polynomials arising in this way. These Garsia numbers have the added property that they are reciprocal mod 2 , in the sense that there exists some $k$ such that $f(z) \equiv z^{k} f^{*}(z)(\bmod 2)$. Let $f(z)=a_{n} z^{n}+\cdots+a_{1} z+a_{0}$ with $a_{n} \neq 0$. Then the reciprocal polynomial $f^{*}(x)$ is defined as $f^{*}(x)=x^{\operatorname{deg}(f)} f(1 / x)=$ $a_{0} z^{n}+a_{1} z^{n-1}+\cdots+a_{n}$. It is easy to verify that $z^{4}+z^{3}-z-2$ is a Garsia polynomial that is not reciprocal mod 2. Hence this construction of McKee and Smyth cannot account for all Garsia numbers. Such examples are not sporadic isolated cases, as the set of positive real Garsia numbers has infinitely many limit points greater than 1 (Theorem 1.2), whereas the real roots of the polynomials of McKee and Smyth have only 1 as a limit point (Lemma 7.1).

The last section, Section 8 discusses some future directions for this work. 


\section{The ALGORIthm}

The algorithm for finding Garsia numbers is closely related to that of Boyd's algorithm for finding Pisot numbers [3, 4]. Since the focus here is just to present some observations, we will omit technical proofs.

2.1. High level description. We start with an algorithm that finds all Garsia numbers that have minimal polynomials of degree at most $n$.

The idea of the algorithm is this. Suppose that $P(z)$ is the minimal polynomial of some Garsia number $\gamma$. We then look at the rational function $P(z) / P^{*}(z)=$ $u_{0}+u_{1} z+u_{2} z^{2}+\cdots$. Since $P(z)$ is Garsia, we can say something about the coefficients of the rational function power series: for example, it is clear that we should have $u_{0}= \pm 2$. In fact, since $P^{*}(0)=1$, it follows that $u_{i} \in \mathbb{Z}$ for all $i$. The interesting thing is that if the coefficients $u_{0}, \ldots, u_{n-1}$ are known, then it is possible to determine finite upper and lower bounds for $u_{n}$.

With that in mind, the most basic form of the algorithm works by building up the sequence $u_{0}, u_{1}, u_{2}, \ldots$, using a tree structure, as follows:

- Each node of the tree represents a subsequence of coefficients $\left[u_{0}, \ldots, u_{n}\right]$, where $n$ is the depth of the node in the tree (using the convention that the root is at depth 0 ).

- The root node contains the coefficient $\left[u_{0}\right]=[2]$.

- For every subsequence of coefficients $\left[u_{0}, \ldots, u_{n}\right]$, we can compute the range of allowable values for $u_{n+1}$. Suppose we find that $A \leq u_{n+1} \leq B$ for some integers $A$ and $B$. Then the children of the node representing $\left[u_{0}, \ldots, u_{n}\right]$ are the nodes representing $\left[u_{0}, \ldots, u_{n}, A\right]$ through $\left[u_{0}, \ldots, u_{n}, B\right]$.

- There is a one-to-one correspondence between the nodes of depth $n$ satisfying a certain condition, described in Section 2.2 and the Garsia numbers of degree $n$.

The algorithm would proceed by building up the tree and checking for nodes that correspond to Garsia numbers. By filling up the tree to depth $n$, the algorithm finds all Garsia numbers of degree less than or equal to $n$. (Note that we enforced that $u_{0}=2$ instead of \pm 2 . This is to ensure that the tree has a single root node. The details of the algorithm make sure that no Garsia numbers are lost by making this restriction.)

It is not difficult to adapt the algorithm so that it finds only the Garsia numbers in a particular interval $[c, d]$. Notice that bounding the range of the Garsia numbers found corresponds to imposing tighter bounds on the coefficients $u_{n}$, given the sequence $\left[u_{0}, \ldots, u_{n-1}\right]$. So, to change the algorithm, we compute the modified coefficient bounds for each node, which will reduce the number of children for some of the nodes in the tree, hence restricting the Garsia numbers that are found.

2.2. The algorithm made precise. The main idea of the algorithm, as described in the previous section, involves building up a tree where each node represents a coefficient sequence $\left[u_{0}, \ldots, u_{n}\right]$. To carry out the algorithm, each node is also equipped with additional information. Given a node at depth $n$ with coefficient sequence $\left[u_{0}, \ldots, u_{n}\right]$, the following pieces of information are stored:

- The sequence $\left[u_{0}, \ldots, u_{n}\right]$ - this is the identifying feature of the node.

- A polynomial $Q_{n+1}(z) \in \mathbb{Q}(z)$, determined by the coefficients $u_{0}, \ldots, u_{n}-$ this is possibly a Garsia polynomial. 
- A polynomial $R_{n+1}(z) \in \mathbb{Q}(z)$, determined by the coefficients $u_{0}, \ldots, u_{n}-$ this is possibly a Garsia polynomial.

- A rational number $v_{n+1}$, determined by $Q_{n+1}$ - this is the lower bound for $u_{n+1}$ that restricts the search to Garsia numbers.

- A rational number $w_{n+1}$, determined by $R_{n+1}$ - this is the upper bound for $u_{n+1}$ that restricts the search to Garsia numbers.

- A real number $V_{n+1}$, determined by $c$, the polynomials $R_{n}(z)$ and $R_{n+1}(z)$, and the values of $w_{n}, w_{n+1}$, and $u_{n}$ - this is the lower bound for $u_{n+1}$ that restricts the search to numbers larger than $c$.

- A real number $W_{n+1}$, determined by $d$, the polynomials $Q_{n}(z)$ and $Q_{n+1}(z)$, and the values of $v_{n}, v_{n+1}$, and $u_{n}$ - this is the upper bound for $u_{n+1}$ that restricts the search to numbers smaller than $d$.

The tree is then built up with nodes storing all of this information, where the children of the node with sequence $\left[u_{0}, \ldots, u_{n}\right]$ will have $u_{n+1} \in\left[\max \left(v_{n+1}, V_{n+1}\right)\right.$, $\left.\min \left(w_{n+1}, W_{n+1}\right)\right] \cap \mathbb{Z}$.

The values listed above are computed using the following recursive formulas:

$$
\begin{aligned}
Q_{n+1}(z) & =(1+z) Q_{n}(z)-\frac{u_{n}-v_{n}}{u_{n-1}-v_{n-1}} z Q_{n-1}(z), \\
R_{n+1}(z) & =(1+z) R_{n}(z)-\frac{u_{n}-w_{n}}{u_{n-1}-w_{n-1}} z R_{n-1}(z), \\
v_{n+1} & =\left[z^{n+1}\right] \frac{Q_{n+1}(z)}{Q_{n+1}^{*}(z)}=\left.\left(\frac{Q_{n+1}(z)-\left(u_{0}+u_{1} z+\cdots+u_{n} z^{n}\right) Q_{n+1}^{*}(z)}{z^{n+1} Q_{n+1}^{*}(z)}\right)\right|_{z=0}, \\
w_{n+1} & =\left[z^{n+1}\right] \frac{-R_{n+1}(z)}{R_{n+1}^{*}(z)}=-\left.\left(\frac{R_{n+1}(z)+\left(u_{0}+u_{1} z+\cdots+u_{n} z^{n}\right) R_{n+1}^{*}(z)}{z^{n+1} R_{n+1}^{*}(z)}\right)\right|_{z=0}, \\
V_{n+1} & =w_{n+1}-\frac{(1+c)^{2}\left(w_{n}-u_{n}\right)\left(u_{n}-V_{n}\right)}{c\left(w_{n}-V_{n}\right)}, \\
W_{n+1} & =v_{n+1}+\frac{(1+d)^{2}\left(W_{n}-u_{n}\right)\left(u_{n}-v_{n}\right)}{d\left(W_{n}-v_{n}\right)} .
\end{aligned}
$$

Here, and throughout this paper, we use the notation that $\left[x^{k}\right] P(x)$ represents the coefficient of $x^{k}$ in the polynomial $P(x)$.

Since the formulas above are recursive, they only apply for $n \geq 1$ (which are at nodes of depth $n-1$, because a node at depth $n$ has the $Q$ polynomial $Q_{n+1}(z)$, etc.). It is necessary to provide some initial base cases, which were computed to be as follows.

For the root node with coefficient sequence $\left[u_{0}\right]=[2]$ :

$$
\begin{aligned}
Q_{1}(z) & =z+2, \\
R_{1}(z) & =z-2, \\
v_{1} & =-3, \\
w_{1} & =3, \\
V_{1} & = \begin{cases}-3 & \text { if } c \leq-2, \\
-2 & \text { if } c>-2,\end{cases} \\
W_{1} & = \begin{cases}2 & \text { if } c<2, \\
3 & \text { if } c \geq 2 .\end{cases}
\end{aligned}
$$


For children of the root node (the nodes at depth of 1 ) with coefficient sequence $\left[u_{0}, u_{1}\right]=\left[2, u_{1}\right]$ :

$$
\begin{aligned}
Q_{2}(z) & =z^{2}-u_{1} z+2, \\
R_{2}(z) & =z^{2}-\frac{u_{1}}{3} z-2 \\
v_{2} & =u_{1}^{2}-3 \\
w_{2} & =\frac{1}{3} u_{1}^{2}+3 \\
V_{2} & =\frac{c u_{1}^{2}-\left(c^{3}+2 c^{2}-c-2\right) u_{1}+3 c^{3}-6}{c(2-c)}, \\
W_{2} & =\frac{d u_{1}^{2}+\left(d^{3}-2 d^{2}-d+2\right) u_{1}+3 d^{3}+6}{d(2+d)} .
\end{aligned}
$$

Once these base cases are established, the recursion formulas described above may be applied, and the tree may be populated and traversed in a straightforward manner.

To identify nodes with their corresponding Garsia polynomials, consider the node with coefficient sequence $\left[u_{0}, \ldots, u_{n}\right]$.

- If $u_{n}=v_{n}$, then $Q_{n}(z)$ is a Garsia polynomial, and this node does not have any children.

- If $u_{n}=w_{n}$, then $R_{n}(z)$ is a Garsia polynomial, and this node does not have any children.

- If neither of the above conditions holds (that is, $v_{n}<u_{n}<w_{n}$ ), then this node does not correspond to a Garsia polynomial.

2.3. The origin of the above formulas. Here is a brief description of how the formulas from the previous section were derived.

Given a sequence of integers $\left[u_{0}, \ldots, u_{n}\right]$, we choose $Q_{n+1}(z)$ to be the unique monic polynomial of degree $n+1$ such that $Q_{n+1}(z) / Q_{n+1}^{*}(z)=u_{0}+u_{1} z+u_{2} z^{2}+$ $\cdots+u_{n} z^{n}+\cdots$, and $R_{n+1}(z)$ to be the unique monic polynomial of degree $n+1$ such that $-R_{n+1}(z) / R_{n+1}^{*}(z)=u_{0}+u_{1} z+u_{2} z^{2}+\cdots+u_{n} z^{n}+\cdots$. The values $v_{n+1}$ and $w_{n+1}$, which bound the possible values of $u_{n+1}$, are found by finding the next coefficient in each of the above power series expansions, so that the following identities hold:

$$
\begin{aligned}
& \frac{Q_{n+1}(z)}{Q_{n+1}^{*}(z)}=u_{0}+u_{1} z+u_{2} z^{2}+\cdots+u_{n} z^{n}+v_{n+1} z^{n+1}+\cdots, \\
& \frac{-R_{n+1}(z)}{R_{n+1}^{*}(z)}=u_{0}+u_{1} z+u_{2} z^{2}+\cdots+u_{n} z^{n}+w_{n+1} z^{n+1}+\cdots .
\end{aligned}
$$

The straightforward approach would be to find the polynomials $Q_{n+1}(z)$ and $R_{n+1}(z)$ by comparing coefficients and solving some linear systems. The values of $v_{n+1}$ and $u_{n+1}$ would then be found by evaluating the coefficient of $z^{n+1}$ in the above series. Rather than carrying out these computations for every node of the tree, it is easier to use the recurrence formulas for $Q_{n+1}(z)$ and $R_{n+1}(z)$ listed earlier, which can be derived using the above definitions for these functions and then finding $v_{n+1}$ and $w_{n+1}$ in the normal way.

It is known [7] that if the $u_{i}$ are chosen so that $v_{n} \leq u_{n} \leq w_{n}$, then the polynomials produced by the algorithm will exactly be the monic, expansive polynomials 
with constant term $u_{0}$. (An expansive polynomial is one whose roots are all outside the unit disc of $\mathbb{C}$.) Since the algorithm starts with $u_{0}=2$, the polynomials will be exactly the Garsia polynomials, as desired.

The technique used to find Garsia numbers within an interval $[c, d]$ requires that there be only one real root within this interval. This will be ensured if we assume $c \geq \sqrt{2}$, as we can have at most one conjugate greater than $\sqrt{2}$. Let $\gamma$ be a Garsia number between $c$ and $d$, and $R_{n}$ and $Q_{n}$ be the polynomials constructed while navigating down the tree towards $\gamma$. Let $R_{n}$ have maximal root $r_{n}$ and $Q_{n}$ have a maximal root $q_{n}$. As with [2, 3, 4] we see that $r_{n}$ and $q_{n}$ form two monotonic sequences approaching $\gamma$ from opposite directions. Further, $r_{n}$ and $q_{n}$ are maximal roots. This translates to the condition:

$$
R_{n}(c) \lim _{z=\infty} R_{n}(z)<0 \text { and } Q_{n}(d) \lim _{z=\infty} Q_{n}(z)>0 .
$$

Further, as $Q_{n}(z)$ and $R_{n}(z)$ are monic, they are both positive as $z$ tends to infinity. This condition is equivalent to $V_{n} \leq u_{n} \leq W_{n}$, as shown in [2, 3, 4].

\section{ISOLATED LIMIT POINTS}

In this section we will show that the root of $z^{2}-z-1$ and $z^{3}-z^{2}-1$ are isolated limit points of the positive real Garsia numbers. Furthermore, we will show that these are the only such limit points greater than $\sqrt{2}$.

We say that $\gamma$ is an isolated limit point if for some $r>0, \gamma$ is the only limit point of the Garsia numbers in the interval $[\gamma-r, \gamma+r]$.

We do this by recalling the general, nonrestricted tree structure used in the algorithm to find Garsia numbers. There is a one-to-one correspondence between leaf nodes in this Garsia search tree and the Garsia numbers in [1,2]. Every branch of the tree is either finite or infinite; the finite ones eventually terminate with a leaf, giving a Garsia number, while the infinite branches do not have leaf nodes of their own, but present infinitely many (potential) branching points that may produce offshoot branches with their own leaf nodes.

If two leaf nodes share many common ancestors in the tree, then the corresponding Garsia numbers will be close together on the real line. Thus, the limit points of Garsia numbers can be interpreted in the tree as follows: if $N_{1}, N_{2}, N_{3}, \ldots$ is a sequence of nodes in the tree such that $N_{i+1}$ is the child of $N_{i}$ for all $i \geq 1$, and for every $m \geq 1$, the node $N_{m}$ has a descendant that is a leaf node, then the branch $N_{1}, N_{2}, N_{3}, \ldots$ corresponds to a limit point of the Garsia numbers. In particular, if the number of such branches beginning with $N_{1}$ is finite, then the limit points corresponding to those branches are isolated.

We will therefore make use of the following definitions:

Definition 3.1. Given a node $N$ in the Garsia search tree, let $T_{N}$ denote the subtree rooted at $N$; that is, the tree consisting of the node $N$ and all descendants of $N$. We will say that the subtree $T_{N}$ contains a limit point if there exists a branch of nodes $N, N_{1}, N_{2}, N_{3}, \ldots$ that corresponds to a limit point of Garsia numbers. If such a branch exists and is unique, then we will say that the subtree $T_{N}$ contains a unique limit point.

It is clear that if a subtree $T_{N}$ contains a unique limit point, then that limit point is isolated. Thus, to find an isolated limit point of the Garsia numbers, it suffices to find a subtree of the Garsia search tree that contains a unique limit point. 
Every node in the Garsia search tree is equipped with two parameters, $v \leq w$, that give bounds on how many children that node can have: the node has exactly one child for every integer $u$ lying within the range $[v, w]$. This motivates the following definition (used by Boyd when describing the analogous tree for Pisot numbers):

Definition 3.2. For a given node on the tree of Garsia numbers described earlier, we say that the width of the node is the value $w-v$.

Thus, if a node has width of $W$, then the node will have at least $\lfloor W\rfloor$ children and at most $\lfloor W\rfloor+1$ children. By the algorithm, the leaf nodes are nodes that have width exactly 0 .

A nontrivial fact is that as we move further down the tree, the widths of nodes decrease. This comes from the identity stated by Boyd, which says that for a node at level $n$ with bounds $v_{n}$ and $w_{n}$,

$$
w_{n+1}-v_{n+1}=\frac{4\left(w_{n}-u_{n}\right)\left(u_{n}-v_{n}\right)}{w_{n}-v_{n}},
$$

where $u_{n}$ is an integer in $\left[v_{n}, w_{n}\right]$ giving rise to a child with bounds $v_{n+1}$ and $w_{n+1}$. It follows that

$$
w_{n+1}-v_{n+1} \leq w_{n}-v_{n},
$$

and hence the width of a child node is never larger than the width of its parent.

A consequence of this decreasing width property is that it is less common for nodes deep down the tree to have many children. For example, once a node has width strictly less than 1 , neither it nor any of its descendants can support more than a single child. Thus, any nonleaf node $N_{0}$ with width less than 1 has two possibilities: either its descendants continue for a finite number of generations in a chain, eventually terminating with a leaf node (and hence a single Garsia number), or its descendants continue for an infinite number of generations to form an infinitely long chain, with no leaf node at all (hence no Garsia numbers). In any case, if the node $N_{0}$ has width less than 1 , then the subtree $T_{N_{0}}$ will contain at most one leaf node, and in particular, $T_{N_{0}}$ will not contain any limit points.

This results in the following useful lemma:

Lemma 3.1. Let $N_{1}, N_{2}, N_{3}, \ldots$ be an infinite sequence of nodes in the Garsia search tree such that the node $N_{i+1}$ is a child of the node $N_{i}$ for all $i \geq 1$. Suppose that for each $i \geq 1$, the node $N_{i}$ has exactly one child having width of at least 1 , namely $N_{i+1}$. Then the subtree rooted at the node $N_{1}$ contains at most one Garsia limit point, and if it exists, this limit point is isolated.

Proof. Note that if the branch $M_{1}, M_{2}, M_{3}, M_{4}, \ldots$ represents a limit point in the subtree $T_{N_{1}}$, where $M_{1}=N_{1}$, then it must be the case that $M_{i}=N_{i}$ for all $i \geq 2$. For otherwise, let $k \geq 1$ be the smallest integer such that $M_{k}=N_{k}$ but $M_{k+1} \neq N_{k+1}$. Then $M_{k+1}$ must have width strictly less than 1 , since $N_{k+1}$ is the only child of $N_{k}$ with width of at least 1 . But this would imply that $T_{M_{k}+1}$ does not contain a limit point, which contradicts the fact that $M_{k+1}$ is a node in a branch representing a limit point. Thus if the subtree $T_{N_{1}}$ contains a limit point, it must contain a unique limit point. Hence $T_{N_{1}}$ contains at most one limit point, which is isolated if it exists.

The next lemma helps identify situations where Lemma 3.1 is applicable. 
Lemma 3.2. If a node $N$ in the Garsia search tree has width less than 2.1 and has a child of width greater than 2 , then $N$ has exactly one child having width of at least 1 .

Proof. Let $N$ be a node in the Garsia search tree. Suppose that $N$ has width $W<2.1$, and that $N$ has at least one child with width greater than 2 . Since the width of a child node is never larger than the width of the parent node, we deduce that $2<W<2.1$, and thus $N$ will either have two children or three children. Without loss of generality, we will assume that the interval corresponding to this width is $\left[v_{n}, w_{n}\right]=[0, W]$.

Suppose that $N$ has exactly three children, corresponding to three integers $u, u+$ $1, u+2$ in the interval $\left[v_{n}, w_{n}\right]=[0, W]$. In order to fit all three integers in this interval, we must have $0 \leq u \leq W-2<0.1$.

The left child, given by the integer $u$, has width

$$
\begin{aligned}
\frac{4 u(W-u)}{W} & =4 u-\frac{4 u^{2}}{W} \\
& \leq 4 u \\
& <4(0.1)<1 .
\end{aligned}
$$

Similarly, the right child of $N$, corresponding to the integer $u+2$, has width

$$
\begin{aligned}
\frac{4(u+2)(W-u-2)}{W} & =4(W-u-2)-\frac{4(W-u-2)^{2}}{W} \\
& \leq 4(W-u-2) \\
& \leq 4(W-2) \\
& <4(0.1)<1 .
\end{aligned}
$$

Thus at least two of the three children of node $N$ have width strictly less than 1. Thus the remaining child must be the one with width greater than 2 , and hence $N$ has only one child with width of at least 1 , as desired.

Suppose now that $N$ has exactly two children, corresponding to two integers $u$ and $u+1$ in the interval $\left[v_{n}, w_{n}\right]=[0, W]$. In order to prevent having more than two integers in this interval, we must have $W-2<u \leq W-2<0.1$.

By hypothesis, at least one of the two children nodes of $N$ has width larger than 2. Without loss of generality, suppose that the left child, corresponding to integer $w$, has width larger than 2. This implies that

$$
2<\frac{4 u(W-u)}{W} .
$$

If the right child of $N$, corresponding to integer $u+1$, has width strictly less than 1, then $N$ has exactly one child with width larger than 1 , and the theorem is proven. We may therefore assume that the right child has width of at least 1; thus we have

$$
1<\frac{4(u+1)(W-u-1)}{W}
$$


Now, equation (2) implies that

$$
\begin{aligned}
2 W & <4 u W-4 u^{2}, \\
0 & >2 u^{2}-2 u W+W
\end{aligned}
$$

and thus

$$
\frac{W}{2}-\frac{\sqrt{W^{2}-2 W}}{2}<u<\frac{W}{2}+\frac{\sqrt{W^{2}-2 W}}{2} .
$$

Similarly, equation (3) implies that

$$
\begin{aligned}
W & \leq 4 u W-4 u^{2}-8 u+4 W-4, \\
0 & \geq 4 u^{2}-(4 W-8) u+(4-3 W),
\end{aligned}
$$

and so we obtain

$$
\frac{W}{2}-1-\frac{\sqrt{W^{2}-W}}{2} \leq u \leq \frac{W}{2}-1+\frac{\sqrt{W^{2}-W}}{2} .
$$

Combining both inequalities, it follows that

$$
\frac{W}{2}-\frac{\sqrt{W^{2}-2 W}}{2}<u \leq \frac{W}{2}-1+\frac{\sqrt{W^{2}-W}}{2},
$$

and in particular, we have

$$
\begin{aligned}
\frac{W}{2}-\frac{\sqrt{W^{2}-2 W}}{2} & <\frac{W}{2}-1+\frac{\sqrt{W^{2}-W}}{2}, \\
\sqrt{W^{2}-2 W} & >2-\sqrt{W^{2}-W}, \\
W^{2}-2 W & >4-4 \sqrt{W^{2}-W}+W^{2}-W, \\
W+4 & <4 \sqrt{W^{2}-W}, \\
W^{2}+8 W+16 & <16 W^{2}-16 W, \\
15 W^{2}-24 W-16 & >0 .
\end{aligned}
$$

Thus, in order for both inequalities (2) and (3) to hold, we must have $15 W^{2}-$ $24 W-16>0$. The roots of the polynomial $15 W^{2}-24 W-16$ are $(4 \pm 8 \sqrt{6}) / 5$, so (2) and (3) imply that either

$$
W<\frac{4-8 \sqrt{6}}{5}<0
$$

or

$$
W>\frac{4+8 \sqrt{6}}{5}>2.1
$$

However, we have $0 \leq W \leq 2.1$, so neither condition is satisfied. Therefore, at least one of the inequalities (2) or (3) must be false.

Thus, if $N$ has exactly two children, one of which has width larger than 2 , then the other must have width strictly less than 1 , which completes the proof of the theorem. 
Note that the statement of the theorem can be improved. Rather than requiring a node to have width less than 2.1, we can relax the requirement to having width less than $(4+8 \sqrt{6}) / 5 \approx 2.1063$. However, this relaxation is not necessary, and makes the statement and proof of the theorem more complicated.

Lemma 3.3. Let $F_{0}=0, F_{1}=1$ and $F_{n}=F_{n-1}+F_{n-2}$ be the standard Fibonacci numbers. Let $T_{0}=0, T_{1}=T_{2}=1$ and $T_{n}=T_{n-1}+T_{n-3}$. Then the nodes

$$
\left[2, F_{3}, F_{4}, F_{5}, F_{6}, \cdots, F_{n}\right] \text { and }\left[2, T_{3}, T_{4}, T_{5}, T_{6}, \cdots, T_{n}\right]
$$

have at least three children, and hence have width at least 2.

Proof. For $n \geq 1$, let $R_{n}(x)=\left(x^{2}-x-1\right) x^{n}+\left(x^{2}-2\right)$. It is known that $R_{n}(x)$ has a Garsia number as a root for all $n \geq 1$.

Let $F_{n}$ be a sequence of integers where $F_{1}=1, F_{2}=2$, and $F_{n}=F_{n-1}+F_{n-2}$ for all $n \geq 3$.

For $n \geq 3$, we will prove that

$$
\frac{R_{n}(x)}{-R_{n}^{*}(x)}=2+\sum_{j=2}^{n} F_{j} x^{j-1}+\sum_{j=1}^{3}\left(F_{n+j}-j\right) x^{n+j-1}+O\left(x^{n+3}\right) .
$$

We will show that

$$
\begin{aligned}
R_{n}(x) & =-R_{n}^{*}(x)\left(2+\sum_{j=2}^{n} F_{j} x^{j-1}+\sum_{j=1}^{3}\left(F_{n+j}-j\right) x^{n+j-1}+O\left(x^{n+3}\right)\right) \\
& =-R_{n}^{*}(x)\left(2+\sum_{j=2}^{n} F_{j} x^{j-1}+\sum_{j=1}^{3}\left(F_{n+j}-j\right) x^{n+j-1}\right)+O\left(x^{n+3}\right)
\end{aligned}
$$

by demonstrating that the coefficients of

$$
\begin{aligned}
f(x) & :=-R_{n}^{*}(x)\left(2+\sum_{j=2}^{n} F_{j} x^{j-1}+\sum_{j=1}^{3}\left(F_{n+j}-j\right) x^{n+j-1}\right) \\
& =\left(2 x^{n+2}-x^{n}+x^{2}+x-1\right)\left(2+\sum_{j=2}^{n} F_{j} x^{j-1}+\sum_{j=1}^{3}\left(F_{n+j}-j\right) x^{n+j-1}\right)
\end{aligned}
$$

are the same as those of $R_{n}(x)=x^{n+2}-x^{n+1}-x^{n}+x^{2}-2$ for all terms of degree up to $n+2$. 
It is straightforward to see that the following equations hold for $n \geq 3$ :

$$
\begin{aligned}
{\left[x^{0}\right] f(x) } & =-2=\left[x^{0}\right] R_{n}(x), \\
{\left[x^{1}\right] f(x) } & =2-F_{2} \\
& =2-2 \\
& =0=\left[x^{1}\right] R_{n}(x), \\
{\left[x^{2}\right] f(x) } & =2+F_{2}-F_{3} \\
& =2+2-3 \\
& =1=\left[x^{2}\right] R_{n}(x), \\
{\left[x^{j}\right] f(x) } & =F_{j-1}+F_{j}-F_{j+1} \\
& =0=\left[x^{j}\right] R_{n}(x) \text { for } j=3, \ldots, n-1, \\
{\left[x^{n}\right] f(x) } & =-2+F_{n-1}+F_{n}-\left(F_{n+1}-1\right) \\
& =-1+\left(F_{n-1}+F_{n}-F_{n+1}\right) \\
& =-1=\left[x^{n}\right] R_{n}(x), \\
{\left[x^{n+1}\right] f(x) } & =-2+F_{n}+\left(F_{n+1}-1\right)-\left(F_{n+2}-2\right) \\
& =-1+\left(F_{n}+F_{n+1}-F_{n+2}\right) \\
& =-1=\left[x^{n+1}\right] R_{n}(x), \\
{\left[x^{n+2}\right] f(x) } & =2(2)-F_{3}+\left(F_{n+1}-1\right)+\left(F_{n+2}-2\right)-\left(F_{n+3}-3\right) \\
& =4-3+\left(F_{n+1}+F_{n+2}-F_{n+3}\right)-1-2+3 \\
& =1=\left[x^{n+2}\right] R_{n}(x) .
\end{aligned}
$$

Hence $f(x)$ and $R_{n}(x)$ have the same coefficients up to degree $n+2$; thus $R_{n}(x)=f(x)+O\left(x^{n+3}\right)$. This completes the proof of the claim.

For $n \geq 2$, let $Q_{n}(x):=\left(x^{2}-x-1\right) x^{n}-\left(x^{2}-2\right)=x^{n+2}-x^{n+1}-x^{n}-x^{2}+2$. It is known that $Q_{n}(x)$ has a Garsia number as a root for all $n \geq 2$.

Using these polynomials, we make the following claim:

$$
\frac{Q_{n}(x)}{Q_{n}^{*}(x)}=2+\sum_{j=2}^{n} F_{j} x^{j-1}+\left(F_{n+1}+1\right) x^{n}+O\left(x^{n+1}\right) .
$$

The proof of this claim is similar to that of the previous claim, where for the $n \geq 3$ case we show the stronger claim that

$$
\frac{Q_{n}(x)}{Q_{n}^{*}(x)}=2+\sum_{j=2}^{n} F_{j} x^{j-1}+\sum_{j=1}^{3}\left(F_{n+j}+j\right) x^{n+j-1}+O\left(x^{n+3}\right) .
$$

The details will not be shown.

Now, for any $n \geq 1$, the coefficients of the series expansions of $R_{n}(x) /\left(-R_{n}^{*}(x)\right)$ and $Q_{n}(x) / Q_{n}^{*}(x)$ agree for the first $n$ terms, as $R_{n}(x) /\left(-R_{n}^{*}(x)\right)$ has the coefficient sequence

$$
\left[2, F_{2}, F_{3}, F_{4}, \ldots, F_{n-1}, F_{n}, F_{n+1}-1, \ldots\right]
$$

and $Q_{n}(x) / Q_{n}^{*}(x)$ has the coefficient sequence

$$
\left[2, F_{2}, F_{3}, F_{4}, \ldots, F_{n-1}, F_{n}, F_{n+1}+1, \ldots\right]
$$


which proves the result.

The proof of the second node is similar to that of the first.

The next Lemma is the result of a direct computation.

Lemma 3.4. Let $F_{n}$ and $T_{n}$ be as before. Then the nodes

$$
\begin{array}{r}
{\left[2, F_{3}, F_{4}, F_{5}, F_{6}, \ldots, F_{41}\right]} \\
{\left[2, T_{3}, T_{4}, T_{5}, T_{6}, \ldots, T_{61}\right]}
\end{array}
$$

have width $2.09545454 \ldots$ and $2.09583333 \ldots$, respectively.

Proof of Theorem 1.1. Let $F_{n}$ and $T_{n}$ be as before. Then by Lemmas 3.2, 3.3, and 3.4 the nodes

$$
\begin{array}{r}
{\left[2, F_{3}, F_{4}, F_{5}, F_{6}, \cdots, F_{41}\right],} \\
{\left[2, T_{3}, T_{4}, T_{5}, T_{6}, \cdots, T_{61}\right]}
\end{array}
$$

are the start of an infinite branch. The main trunk of this branch is made up of nodes of the form $\left[2, F_{3}, F_{4}, F_{5}, F_{6}, \ldots, F_{n}\right]$ and $\left[2, T_{3}, T_{4}, T_{5}, T_{6}, \ldots, T_{n}\right]$. From each of these nodes, there are three children. The middle child is of the form $\left[2, F_{3}, F_{4}, F_{5}, F_{6}, \ldots, F_{n+1}\right]$ and $\left[2, T_{3}, T_{4}, T_{5}, T_{6}, \ldots, T_{n+1}\right]$, and has width greater than 2 . The two other children are of the form $\left[2, F_{3}, F_{4}, F_{5}, F_{6}, \ldots, F_{n+1} \pm 1\right]$ and $\left[2, T_{3}, T_{4}, T_{5}, T_{6}, \ldots, T_{n+1} \pm 1\right]$ having width less than 1 . These two other children are associated with the Garsia numbers

$$
\left(z^{2}-z-1\right) z^{k} \pm\left(z^{2}-2\right) \text { and }\left(z^{3}-z^{2}-1\right) z^{k} \pm\left(z^{3}-z^{2}+z-2\right),
$$

whereas the middle child is associated to the limit point $z^{2}-z-1$ and $z^{3}-z^{2}-1$. See Figure 1 below.

A quick check of all Garsia numbers in $[\sqrt{2}, 2]$ shows that these are the only nodes of degree greater than 60 with width greater than 1 . This completes the proof.

\section{A construction using Rouche's Theorem}

In the previous section we showed the existence of two isolated limit points. In this section, we give sufficient criteria for a limit point to exist, as well as give an infinite family of limit points of Garsia numbers.

Lemma 4.1. Consider a pair of polynomials $f(z)$ and $g(z)$ with the following properties:

(1) $f(z)$ is monic.

(2) $g(z)$ is the minimal polynomial of a complex Garsia number.

(3) $|f(z)| \leq|g(z)|$ on $|z|=1$.

Then

$$
f(z) z^{k} \pm g(z)
$$

has a Garsia number as a root.

Proof. By Rouche's Theorem, (4) will have no roots strictly inside the unit circle. For $k \geq 1$, this polynomial will be of norm \pm 2 , and hence it will have a (possibly complex) Garsia number as a root. If $f(z)$ has a root $|\gamma|>1$, then one of the roots of $f(z) z^{k} \pm g(z)$ will approach $\gamma$ as $k \rightarrow \infty$. 


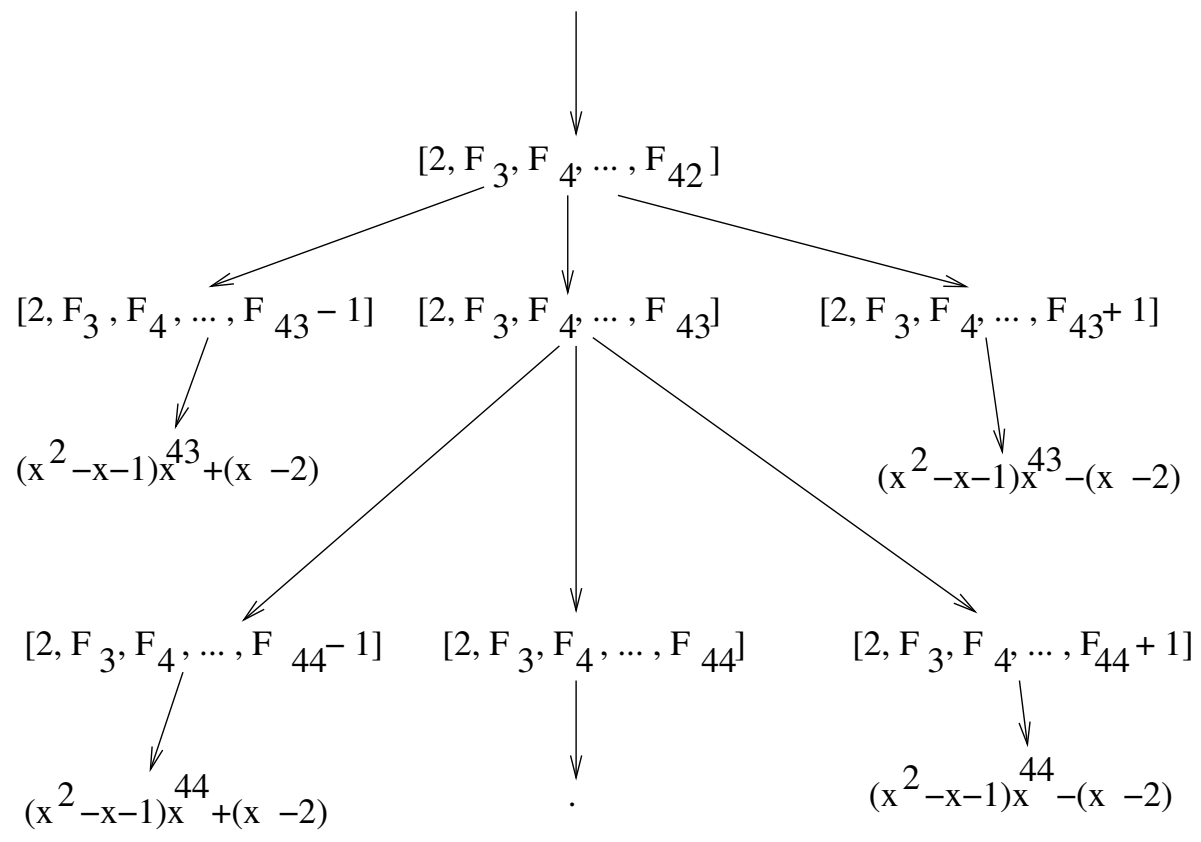

Figure 1. Tree for limit points associated with $z^{2}-z-1$.

Consider as an example:

$$
\left(z^{2}-z-1\right) z^{k} \pm\left(z^{2}-2\right)
$$

The roots of these polynomials have the golden ratio as a limit point as $k$ tends to infinity. Combining this with Lemma 1.1 gives an infinite number of limit points, with these limit points tending to 1 . Hence 1 is in the second derived set of Garsia numbers.

Based upon some computational searches (discussed in Section 6), it is believed that all limit points will be of this form.

Proof of Theorem 1.2. Let $f(z)=z^{n}-z^{m}-1$ and $g(z)=z^{n}-z^{m}+z^{n-m}-2$. Our claim is that these two polynomials satisfy the conditions of Lemma 4.1] and hence

$$
f(z) z^{k}+g(z)
$$

has a Garsia number as a root.

First, write $z=\cos (\theta)+i \sin (\theta)$.

To show part (3) of Lemma 4.1, it suffices to show that

$$
|g(z)|^{2}-|f(z)|^{2} \geq 0
$$


We notice that

$$
\begin{aligned}
|g(z)|^{2}- & |f(z)|^{2} \\
= & g(z) \overline{g(z)}-f(z) \overline{f(z)} \\
= & (7+6 \cos (m \theta)-4 \cos (n \theta)-6 \cos ((n-m) \theta)-2 \cos ((2 m-n) \theta)) \\
& -(3+2 \cos (m \theta)-2 \cos (n \theta)-2 \cos ((n-m) \theta)) \\
= & 4+4 \cos (m \theta)-4 \cos ((n-m) \theta)-2 \cos ((m-n) \theta-m \theta) \\
& -2 \cos ((m-n) \theta+m \theta) \\
= & 4+4 \cos (m \theta)-4 \cos ((n-m) \theta)-4 \cos ((m-n) \theta) \cos (m \theta) \\
= & 4(1+\cos (m \theta))(1-\cos ((m-n) \theta)) \\
\geq & 0
\end{aligned}
$$

as each individual factor is greater than or equal to 0 .

To see that $z^{n}-z^{m}+z^{n-m}-2$ has a Garsia root, we apply Rouche's theorem to the two polynomials $f_{1}(z)=z^{n}+z^{n-m}$ and $g_{1}(z)=z^{m}+2$. Clearly, $\left|f_{1}(z)\right|=$ $\left|z^{n}+z^{n-m}\right|=\left|z^{n-m}\right| \cdot\left|z^{m}+1\right|=\left|z^{m}+1\right|$ and $\left|g_{1}(z)\right|=\left|z^{m}+2\right|$. We see that the imaginary part of $z^{m}+1$ is the same as that for $z^{m}+2$, and the real part of $z^{m}+1$ is always strictly less than that for $z^{m}+2$. Hence $\left|f_{1}(z)\right|<\left|g_{1}(z)\right|$ on the unit circle, and so $f_{1}(z)+g_{1}(z)=z^{n}-z^{m}+z^{n-m}-2$ is a Garsia polynomial, as required.

If Conjectures 1.1 and 1.2 are true, then all limit points will be of this form. This, combined with the lemma below, would show that the set of positive real Garsia numbers is nowhere dense.

Lemma 4.2. The set of limit points of the real roots of $z^{n}-z^{m}-1$ is the singleton $\{1\}$.

Proof. Let $\lambda_{n, k}$ be the root of $z^{n+k}-z^{n}-1$. This is equivalent to $z^{k}=1+1 / z^{n} \leq 2$, which implies that $\lambda_{n, k} \leq \sqrt[k]{2}$. This, combined with the fact that for fixed $k$, $\lim _{n \rightarrow \infty} \lambda_{n, k}=1$, gives the desired result.

Recall that for Pisot numbers, there is a nice construction for all of the limit points. Moreover, we know the families of Pisot numbers that approach these limit points. These limit points, along with the Pisot numbers approaching them, are called regular Pisot numbers. Inspired by this notation, we say that the Garsia numbers arising from equation 4 , where $f(z)$ has a positive real root greater than 1 , are regular Garsia numbers. Ideally, we would like to say that these are equivalent to Garsia numbers arising from equation (11). Unfortunately, we do not know that all roots look like roots of $z^{n}-z^{m}-1$. Moreover, even if we did know that, we do not know that all of the companion Garsia polynomials needed for this result are of the form $z^{n}-z^{m}+z^{n-m}+2$.

\section{Irregular Garsia numbers exist}

For this section, we are assuming that all regular Garsia numbers are the roots of polynomials of the form $f(z) z^{k} \pm g(z)$, where $f(z)=z^{n}-z^{m}-1$ and $g(z)=$ $z^{n}-z^{m}+z^{n-m}-2$ for some $n>m \geq 1$ and $k \geq 1$. 
TABLE 1. Irregular Garsia numbers greater than $\sqrt{2}$, with degree less than or equal to 10

\begin{tabular}{ll}
\hline Minimal polynomial & Root \\
\hline$z^{7}-z^{6}+z^{4}-2 z^{3}-2$ & 1.429566834 \\
$z^{7}-2 z^{6}+z^{5}-z^{2}+2 z-2$ & 1.439412380 \\
$z^{10}-z^{9}-z^{6}-z^{2}+z-2$ & 1.437256211 \\
$z^{10}-z^{9}-z^{8}+z^{7}-z^{6}+z^{4}-z^{3}+z^{2}+z-2$ & 1.440735756 \\
$z^{9}-z^{8}-z^{7}+z^{4}+z^{2}-2$ & 1.458430012 \\
$z^{7}-2 z^{5}-z^{4}+2 z^{3}+z^{2}-2 z-2$ & 1.469645598 \\
$z^{9}-2 z^{8}+z^{7}-z^{5}+z^{4}-z^{2}+2 z-2$ & 1.477445065 \\
$z^{9}-z^{7}-z^{6}-z^{4}-z-2$ & 1.483479402 \\
$z^{8}-z^{7}-z^{5}-z^{4}+z^{2}+2$ & $1.144777447,1.493295249$ \\
$z^{7}-z^{6}-z^{5}-z^{4}+2 z^{3}+z^{2}-2$ & 1.496662996 \\
$z^{6}-2 z^{5}+z^{4}-z^{2}+2 z-2$ & 1.497886622 \\
$z^{9}-2 z^{7}-2 z^{6}+2 z^{4}+3 z^{3}+z^{2}-2 z-2$ & 1.529496606 \\
$z^{9}-z^{8}-z^{7}+z^{6}-z^{4}-z^{3}+z^{2}-2$ & 1.522635880 \\
$z^{9}-z^{8}-z^{5}-z^{3}-z^{2}-2$ & 1.535063780 \\
$z^{5}-z^{4}-2 z^{3}+2 z^{2}+z-2$ & 1.537518976 \\
$z^{10}-z^{9}-z^{7}-z^{6}+z^{5}-z^{4}+z^{2}+2$ & $1.090416639,1.542206131$ \\
$z^{8}-z^{6}-2 z^{5}-z^{4}-z^{3}+z^{2}+2 z+2$ & $1.082409688,1.559601910$ \\
$z^{10}-2 z^{9}+z^{8}-z^{7}+z^{6}-z^{4}+z^{3}-z^{2}+2 z-2$ & 1.571572261 \\
$z^{4}-2 z^{3}+2 z-2$ & 1.716672749 \\
\hline
\end{tabular}

As predicted, it is true that not all Garsia numbers follow a "regular" pattern. Assuming Conjecture 1.1 and 1.2, then there exist irregular Garsia numbers.

Theorem 5.1. There exist Garsia numbers whose minimal polynomial does not divide

$$
\left(z^{n}-z^{m}-1\right) z^{k} \pm\left(z^{n}-z^{m}+z^{n-m}-2\right)
$$

for any values of $n>m \geq 1$ and $k \geq 1$.

Some examples of such numbers are given in Table 1

There are 61 irregular Garsia numbers greater than $\sqrt{2}$. It appears that there are an infinite number of irregular Garsia numbers, although the only limit point of irregular Garsia numbers appears to be 1 (see Section [6).

To prove the theorem, we will need to first establish a few lemmas. The first lemma tells us from which side each regular Garsia polynomial approaches its corresponding limit point.

Lemma 5.1. Let $\lambda=\lambda_{n, m}>1$ be the positive real root of $f(z)=f_{n, m}(z)=$ $z^{n}-z^{m}-1$, and let $g(z)=g_{n, m}(z)=z^{n}-z^{m}+z^{n-m}-2$. Then as $k$ approaches infinity, the Garsia root of $A(z)=A_{n, m, k}(z)=f(z) z^{k}+g(z)$ approaches $\lambda$ from below, and the Garsia root of $B(z)=B_{n, m, k}=f(z) z^{k}-g(z)$ approaches $\lambda$ from above.

Proof. The positive, real root of $f(z) z^{k} \pm g(z)$ approaches $\lambda$ as $k$ approaches infinity. It remains to prove that the limit is approached from below when the "+" is used, and from above when the " - " is used. We use the fact that in the neighbourhood 
around $\lambda$, the roots of $A(z)$ and $B(z)$ are isolated, and both functions change sign from negative to positive at their roots.

Note that

$$
\begin{aligned}
A(\lambda) & =f(\lambda) \lambda^{k}+g(\lambda) \\
& =0+\left(\left(\lambda^{m}-\lambda^{n}\right)+\lambda^{m-n}-2\right) \\
& =1+\lambda^{m-n}-2 \\
& =\lambda^{m-n}-1>0 .
\end{aligned}
$$

Since $A(\lambda)$ is positive, the Garsia root of $A(z)$ must be to the left of $\lambda$ along the $x$-axis, and hence the root of $A(z)$ approaches $\lambda$ from below.

A similar argument shows that the Garsia root of $B(z)$ approaches $\lambda$ from above, as desired.

The next lemma shows that the Garsia roots of sequences of regular Garsia polynomials approach their limit points monotonically.

Lemma 5.2. Using the notation from Lemma 5.1, let $\alpha_{k}=\alpha_{n, m, k}$ be the Garsia root of $A_{n, m, k}(z)$, and let $\beta_{k}=\beta_{n, m, k}$ be the Garsia root of $B_{n, m, k}(z)$. Then as $k$ increases, $\alpha_{k}$ is strictly increasing and $\beta_{k}$ is strictly decreasing.

Proof. Since $A_{n, m, k}\left(\alpha_{k}\right)=0$, we have that

$$
\left(\alpha_{k}^{m}-\alpha_{k}^{n}-1\right) \alpha_{k}^{k}+\left(\alpha_{k}^{m}-\alpha_{k}^{n}+\alpha_{k}^{m-n}-2\right)=0,
$$

or equivalently

$$
\left(\alpha_{k}^{m}-\alpha_{k}^{n}-1\right) \alpha_{k}^{k}=-\left(\alpha_{k}^{m}-\alpha_{k}^{n}+\alpha_{k}^{m-n}-2\right)<0 .
$$

Here, both sides of the equality are negative, since $f_{n, m, k}\left(\alpha_{k}\right)=\alpha_{k}^{m}-\alpha_{k}^{n}-1<0$ as $\alpha_{k}>1$ is less than the root of $f_{n, m, k}(z)$.

Multiplying by $\alpha_{k}$ gives

$$
\begin{aligned}
\left(\alpha_{k}^{m}-\alpha_{k}^{n}-1\right) \alpha_{k}^{k+1} & =-\left(\alpha_{k}^{m}-\alpha_{k}^{n}+\alpha_{k}^{m-n}-2\right) \alpha_{k} \\
& <-\left(\alpha_{k}^{m}-\alpha_{k}^{n}+\alpha_{k}^{m-n}-2\right)
\end{aligned}
$$

since $\alpha_{k}>1$, and so

$$
\left(\alpha_{k}^{m}-\alpha_{k}^{n}-1\right) \alpha_{k}^{k+1}+\left(\alpha_{k}^{m}-\alpha_{k}^{n}+\alpha_{k}^{m-n}-2\right)<0 .
$$

Thus $\alpha_{k}$ is strictly to the left of $\alpha_{k+1}$, the root of $f(z) z^{k+1}+g(z)$. This means that $\alpha_{1}<\alpha_{2}<\alpha_{3}<\cdots<\lambda_{n, m, k}$.

A similar argument shows that as $k$ increases, then the $\beta_{k}$ decrease.

Our next lemma shows that as the parameter $m$ increases in a regular Garsia polynomial, the Garsia number moves farther from the limit point.

Lemma 5.3. Using the notation from the previous lemma, we let $\beta_{m}=\beta_{n, m, k}$ be the Garsia root of $B_{n, m, k}(z)$. Then for fixed $n$ and fixed $k$, as $m$ increases from 1 to $n-1$, we have that $\beta_{m}$ is strictly increasing. Similarly, $\alpha_{m}$ is strictly decreasing.

Proof. We are given that

$$
\left(\beta_{m}^{n}-\beta_{m}^{m}-1\right) \beta_{m}^{k}-\left(\beta_{m}^{n}-\beta_{m}^{m}+\beta_{m}^{n-m}-2\right)=0,
$$

or equivalently

$$
\beta_{m}^{m}\left(\beta_{m}^{k}-1\right)+\beta_{m}^{n-m}=\beta_{m}^{n+k}-\beta_{m}^{n}-\beta_{m}^{k}+2 .
$$


Consider the left side of this equation. If we increase $m$ by 1 , then the value of the left side of the equation increases; this can be seen by noting that

$$
\begin{gathered}
\left(\beta_{m}^{m}\left(\beta_{m}^{k}-1\right)-\beta_{m}^{n-m}\right)-\left(\beta_{m}^{m+1}\left(\beta_{m}^{k}-1\right)-\beta_{m}^{n-(m+1)}\right) \\
=\left(1-\beta_{m}\right)\left(\beta_{m}^{m}\left(\beta_{m}^{k}-1\right)+\beta_{m}^{n-m-1}\right)
\end{gathered}
$$

is negative since $\beta_{m}>1$.

Hence

$$
\begin{aligned}
\beta_{m}^{m+1}\left(\beta_{m}^{k}-1\right)-\beta_{m}^{n-(m+1)} & >\beta_{m}^{m}\left(\beta_{m}^{k}-1\right)-\beta_{m}^{n-m} \\
& =\beta_{m}^{n+k}-\beta_{m}^{n}-\beta_{m}^{k}+2
\end{aligned}
$$

so

$$
\begin{aligned}
0 & <\beta_{m}^{m+1}\left(\beta_{m}^{k}-1\right)-\beta_{m}^{n-(m+1)}-\beta_{m}^{n+k}+\beta_{m}^{n}+\beta_{m}^{k}-2 \\
& <-\beta_{m}^{n+k}+\beta_{m}^{m+1+k}+\beta_{m}^{k}+\beta_{m}^{n}-\beta_{m}^{m+1}-\beta_{m}^{n-(m+1)}-2 \\
& <\left(\beta_{m}^{n}-\beta_{m}^{m+1}-1\right) \beta_{m}^{k}-\left(\beta_{m}^{n}-\beta_{m}^{m+1}+\beta_{m}^{n-(m+1)}+2\right)
\end{aligned}
$$

and thus the root, $\beta_{m+1}$, of the above polynomial is greater than $\beta_{m}$. Hence $\beta_{n, m, k}<\beta_{n, m+1, k}$ as required.

Combining our results so far gives the next lemma:

Lemma 5.4. For fixed $n$, then $\alpha_{n, n-1,1} \leq \alpha_{n, m, k}<\lambda_{n, m}<\beta n, m, k \leq \beta_{n, n-1,1}$ for all $m$ and $k$.

Proof. We see that for fixed $n$ and $m$ that $\alpha_{n, m, k}$ is minimized and $\beta_{n, m, k}$ is maximized for $k=1$. For fixed $n$ and $k$ we see that $\alpha_{n, m, k}$ is minimized and $\beta_{n, m, k}$ is maximized for $m=n-1$.

We now show that as $m$ increases, the Garsia roots are (in some sense) decreasing.

Lemma 5.5. Let $\hat{\beta}_{n}=\beta_{n, n-1,1}$ be the upper bound for $\beta_{n, m, k}$ for fixed $n$. Then $\hat{\beta}_{n+1}<\hat{\beta}_{n}$.

Proof. From Lemma 5.4, for any given $n \geq 2$, we know that $\hat{\beta}_{n}$ satisfies

$$
\left(\hat{\beta}_{n}^{n}-\hat{\beta}_{n}^{n-1}-1\right) \hat{\beta}_{n}-\left(\hat{\beta}_{n}^{n}-\hat{\beta}_{n}^{n-1}+\hat{\beta}_{n}-2\right)=0 .
$$

We then see that

$$
\hat{\beta}_{n}^{n+1}-2 \hat{\beta}_{n}^{n}+\hat{\beta}_{n}^{n-1}=2 \hat{\beta}_{n}-2>0
$$

and so

$$
\begin{gathered}
\hat{\beta}_{n}\left(\hat{\beta}_{n}^{n+1}-2 \hat{\beta}_{n}^{n}+\hat{\beta}_{n}^{n-1}\right)>\hat{\beta}_{n}^{n+1}-2 \hat{\beta}_{n}^{n}+\hat{\beta}_{n}^{n-1} \\
\hat{\beta}_{n}^{n+2}-2 \hat{\beta}_{n}^{n+1}+\hat{\beta}_{n}^{n}>2 \hat{\beta}_{n}-2 .
\end{gathered}
$$

That is,

$$
\left(\hat{\beta}_{n}^{(n+1)+1}-\hat{\beta}_{n}^{(n+1)}-1\right) \hat{\beta}_{n}-\left(\hat{\beta}_{n}^{(n+1)+1}-\hat{\beta}_{n}^{(n+1)}+\hat{\beta}_{n}-2\right)>0 .
$$

But

$$
\left(\hat{\beta}_{n+1}^{(n+1)+1}-\hat{\beta}_{n+1}^{(n+1)}-1\right) \hat{\beta}_{n+1}-\left(\hat{\beta}_{n+1}^{(n+1)+1}-\hat{\beta}_{n+1}^{(n+1)}+\hat{\beta}_{n+1}-2\right)=0 .
$$


Since the general function

$$
f(z)=\left(z^{(n+1)+1}-z^{(n+1)}-1\right) z-\left(z^{(n+1)+1}-z^{(n+1)}+z-2\right)
$$

is increasing, we can deduce from the above equations and inequalities that $\hat{\beta}_{n+1}<$ $\hat{\beta}_{n}$, and so $\hat{\beta}_{n}$ decreases as $n$ increases, as desired.

We are now ready to prove the main theorem.

Proof of Theorem 5.1. Consider a Garsia number $\gamma$ that we suspect is irregular. We see that there are only a finite number of possible $n$ such that $\alpha_{n, m, k}$ or $\beta_{n, m, k}$ could equal $\gamma$. This is because $\alpha_{n, m, k}, \beta_{n, m, k}<\hat{\beta}_{n}$ and $\hat{\beta}_{n}$ is decreasing. So, we need only check those $n$ such that $\hat{\beta}_{n} \geq \gamma$.

We see that for fixed $n$, there are only a finite number of possible $m$ that need to be checked, namely $m=1,2, \ldots, n-1$.

For fixed $n$ and $m$, we see that we need only check one of $\alpha_{n, m, k}$ or $\beta_{n, m, k}$, but not both. In particular, if $\gamma<\lambda_{n, m}$, we need only check $\alpha_{n, m, k}$; similarly, if $\gamma>\lambda_{n, m}$, we need only check $\beta_{n, m, k}$.

Lastly, for fixed $n$ and $m$, we need only check a finite number of $k$. For example, if $\gamma<\lambda_{n, m}$, we need only check those $k$ such that $\alpha_{n, m, k} \leq \gamma$. Similarly, if $\gamma>\lambda_{n, m}$, we need only check those $k$ such that $\beta_{n, m, k} \geq \gamma$.

Hence for any $\gamma$, we need only check a finite number of regular Garsia numbers to see if $\gamma$ is regular or irregular.

Some examples are summarized in Table 1.

\section{HEURISTIC SEARCH FOR LIMIT POINTS}

We saw in Theorem 1.2 that we have limit points at the roots $\lambda_{n, m}$ of $z^{n}-z^{m}-1$. We also know from Section 3 that the only limit points of positive real Garsia numbers greater than $\sqrt{2}$ are limit points of this type. In this section, we provide some computational evidence that these are the only limit points greater than 1 .

We see from Lemma 4.1 that if we have a (possibly complex) Garsia number with minimal polynomial $g(z)$, and some other polynomial $f(z)$ where $|f(z)| \leq|g(z)|$ on $|z|=1$, then we can use this to construct new Garsia numbers. Moreover, if $f(z)$ has a positive root greater than 1 , then these polynomials have a root approaching the root of $f(z)$.

There are 244 minimal polynomials of Garsia numbers of degree less than or equal to 6 . Call this set $G$. There are are 5229043 monic polynomials of degree less than or equal to 6 with integer coefficients less than 6 in absolute value. Call this set $F$.

For each $f$ in $F$, and each $g$ in $G$, we check if $|f(z)| \leq|g(z)|$ for all $|z|=1$. To perform this computation efficiently, we loop through the possible polynomials in $G$, and for each $g \in G$, we check for each $f \in F$ if the inequality holds at a number of random points around $|z|=1$. Only after we have reduced this list of potential polynomials in $F$ down to a reasonable size (typically, fewer than 100) do we computationally check if the desired inequality holds. To do this, we check if $\left|f\left(x+i \sqrt{1-x^{2}}\right)\right|^{2}-\left|g\left(x+i \sqrt{1-x^{2}}\right)\right|^{2}$ is nonnegative on the interval $[-1,1]$. This is quite easy to automate with a computer.

The results of this search are listed in Table 2. Only those polynomials $f(z)$ such that $f(z)$ has a positive root greater than 1 are listed, although many more can be found by this method. 
TABLE 2. Result of heuristic search of Garsia pairs

\begin{tabular}{lll}
\hline Root of limit point $f(z)$ & $f(z)$ & $g(z)$ \\
\hline 1.134724138 & $z^{6}-z-1$ & $z^{6}+z^{5}-z-2$ \\
1.150963925 & $z^{6}-z^{2}-1$ & $z^{6}+z^{4}-z^{2}-2$ \\
1.167303978 & $z^{5}-z-1$ & $z^{5}+z^{4}-z-2$ \\
1.173984997 & $z^{6}-z^{3}-1$ & $z^{6}-2$ \\
1.193859111 & $z^{5}-z^{2}-1$ & $z^{5}+z^{3}-z^{2}-2$ \\
1.210607794 & $z^{6}-z^{4}-1$ & $z^{6}-z^{4}+z^{2}-2$ \\
1.220744085 & $z^{4}-z-1$ & $z^{4}+z^{3}-z-2$ \\
1.236505703 & $z^{5}-z^{3}-1$ & $z^{5}-z^{3}+z^{2}-2$ \\
1.272019650 & $z^{4}-z^{2}-1$ & $z^{4}-2$ \\
1.285199033 & $z^{6}-z^{5}-1$ & $z^{6}-z^{5}+z-2$ \\
1.324717957 & $z^{5}-z^{4}-1$ & $z^{5}-z^{4}+z-2$ \\
1.324717957 & $z^{3}-z-1$ & $z^{3}+z^{2}-z-2$ \\
1.380277569 & $z^{4}-z^{3}-1$ & $z^{4}-z^{3}+z-2$ \\
1.465571232 & $z^{3}-z^{2}-1$ & $z^{3}-z^{2}+z-2$ \\
1.618033989 & $z^{2}-z-1$ & $z^{2}-2$ \\
\hline
\end{tabular}

This search was repeated with:

- degree 7 polynomials, with $f(z)$ having height at most 4 ,

- degree 8 polynomials, with $f(z)$ having height at most 3,

- degree 9 polynomials, with $f(z)$ having height at most 2,

- degree 14 polynomials, with $f(z)$ having height at most 1 .

No limit points were found that were not predicted by Conjectures 1.1 and 1.2

\section{A construction of McKee and Smyth}

There was a nice observation of McKee and Smyth 14, which can be rearranged for our purposes.

Theorem 7.1. Let $p(z)$ and $q(z)$ be a product of cyclotomic polynomials, $p(0)=$ $q(0)$, and $g(z)=p(z)+q(z)$ a monic polynomial. Then $g(z)$ is a complex Garsia polynomial if and only if $p(z)$ and $q(z)$ have the same degree, no double roots, and their roots on the unit circle interlace.

An easy example of this is $z^{2}-1$ and $-z^{2}+z-1$. We see that their roots interlace, and that their sum is $z-2$, which clearly has the Garsia root 2 .

What is nice about this is that McKee and Smyth give a complete description of all $p(z)$ and $q(z)$ that have this interlacing property. In particular, there are two

TABLE 3. Families of Garsia numbers coming from cyclotomic differences

\begin{tabular}{ll}
\hline$p(z) \quad q(z)$ & Garsia polynomial \\
\hline$\frac{z^{n+1}-1}{z-1} \quad-\frac{\left(z^{k}-1\right)\left(z^{n+1-k}-1\right)}{z-1}$ & $\frac{z^{n+1-k}+z^{k}-2}{z-1}$ \\
$n \geq 1, \operatorname{gcd}(n+1, k)=1,1 \leq k \leq(n+1) / 2$ & \\
$z^{n}+1 \quad-\left(z^{n-k}+1\right)\left(z^{k}-1\right)$ & $z^{n-k}-z^{k}+2$ \\
$n \geq 3, \operatorname{gcd}(k, 2 n)=1,1 \leq k<n$ & \\
\hline
\end{tabular}


TABLE 4. Sporadic examples of complex Garsia polynomials

\begin{tabular}{llll}
\hline$p(z)$ & $q(z)$ & Garsia polynomial & Real Root(s) \\
\hline 12 & $1,2,3$ & $z^{3}+z^{2}-z-2$ & 1.205569430 \\
9 & $1,2,5$ & $z^{5}-z^{3}-z-2$ & 1.455945432 \\
9 & $1,2,4,6$ & $z^{5}-z^{4}+z^{3}+z^{2}-z+2$ & -1.092591837 \\
9 & $1,2,8$ & $z^{4}+z^{3}-z^{2}+2$ & \\
3,12 & $1,2,8$ & $z^{5}+z^{4}-z^{3}-z^{2}+z+2$ & -1.373837398 \\
3,12 & $1,2,5$ & $z^{3}-2 z-2$ & 1.769292354 \\
2,14 & $1,3,5$ & $z^{6}+z^{5}-z^{2}-z-2$ & $-1.433300420,1.158295133$ \\
2,14 & $1,3,12$ & $z^{5}+z^{4}-z^{3}-z^{2}+2$ & -1.564477141 \\
2,18 & $1,3,5$ & $z^{5}+z^{4}+z^{3}-z^{2}-2 z-2$ & 1.172622951 \\
2,18 & $1,3,12$ & $z^{6}+z^{5}-2 z^{3}-z^{2}+z+2$ & \\
2,18 & 1,7 & $z^{6}-z^{4}-z^{3}+z+2$ & \\
15 & $1,2,4,12$ & $z^{7}-z^{6}-z^{5}+z^{4}-z^{3}+z^{2}+z-2$ & 1.390688470 \\
15 & $1,2,14$ & $z^{5}-z^{4}+z^{3}-2 z+2$ & -1.085235385 \\
15 & $1,2,6,10$ & $z^{7}-2 z^{6}+2 z^{5}-z^{4}+2 z^{2}-3 z+2$ & -1.034802737 \\
15 & $1,2,4,10$ & $z^{6}-2 z^{5}+z^{4}-z^{2}+2 z-2$ & $-1.054588290,1.497886622$ \\
15 & $1,2,6,8$ & $z^{4}-2 z^{3}+2 z-2$ & $-1.106919340,1.716672749$ \\
15 & $1,2,6,12$ & $z^{6}-z^{5}-z^{4}+3 z^{3}-z^{2}-2 z+2$ & \\
15 & $1,2,9$ & $z^{7}-z^{6}+z^{4}-2 z^{3}+z^{2}+z-2$ & 1.217967903 \\
15 & $1,2,4,8$ & $z^{7}-z^{5}+z^{4}-z^{3}+z-2$ & 1.147443856 \\
20 & $1,2,3,8$ & $z^{7}+z^{6}-z^{5}-z^{4}+z^{3}+z^{2}-z-2$ & 1.093812686 \\
20 & $1,2,3,12$ & $z^{7}-2 z^{5}-z^{4}+2 z^{3}+2 z^{2}-z-2$ & 1.249400261 \\
20 & $1,2,7$ & $z^{7}+z^{6}-z^{4}+z^{2}-z-2$ & 1.078333775 \\
20 & $1,2,9$ & $z^{5}-z^{4}-z^{3}+2 z^{2}-2$ & 1.292825761 \\
24 & $1,2,4,5$ & $z^{7}+z^{6}+z^{5}+z^{4}-z^{3}-z^{2}-z-2$ & 1.053303524 \\
24 & $1,2,7$ & $z^{7}+z^{4}-z-2$ & 1.080375088 \\
24 & $1,2,9$ & $z^{6}-z^{5}-z^{4}+z^{3}-z^{2}+2$ & \\
\hline & & &
\end{tabular}

infinite families of interlacing $p(z)$ and $q(z)$, and 26 sporadic examples. We repeat them here for completeness. In Table 3 we list the infinite examples. In Table 4 we give the 26 sporadic examples.

It should be noted that not all Garsia polynomials can be written in this way, and further that not all $p(z), q(z)$ with the desired properties have $p(z)-q(z)$ monic. In particular, we have the following:

Lemma 7.1. Let $G$ be the set of positive real Garsia numbers that have minimal polynomial $g(z)=p(z)+q(z)$, where $p(z)$ and $q(z)$ are products of cyclotomic polynomials. Then the only limit point of $G$ is 1 .

Proof. The only limit point coming from the 26 sporadic examples is 1 , which comes from looking at $g\left(z^{n}\right)$ for $g(z)$ a fixed polynomial.

It suffices then to look at the two infinite families. Consider the more general family $z^{n+k} \pm z^{k} \pm 2$. Consider a root $z:=z_{n, k}$ of this polynomial with $|z|>1$. We see that $\left|z^{n}\right| \leq 1+\left|\frac{2}{z^{k}}\right|$. Hence for fixed $k$, we see that $z_{n, k}$ tends to 1 as $n$ tends to infinity. Similarly, for fixed $n$, we see that $z_{n, k}$ tends to 1 as $k$ tends to infinity. As both of these infinite families are subsets of this example, this proves the result. 
TABle 5. Positive real Garsia numbers with degree less than or equal to 6

\begin{tabular}{|c|c|c|c|}
\hline Minimal polynomial & Garsia root & Minimal polynomial & Garsia root \\
\hline$z^{6}+2 z^{5}+z^{4}-z^{2}-2 z-2$ & 1.054588290 & $z^{5}+z^{4}-z^{2}-2 z-2$ & 1.284490835 \\
\hline$z^{6}+z^{5}+z^{4}-z^{2}-z-2$ & 1.070049124 & $z^{5}-z^{4}-z^{3}+2 z^{2}-2$ & 1.292825761 \\
\hline$z^{5}+2 z^{4}+z^{3}-z^{2}-2 z-2$ & 1.072272815 & $z^{5}-z^{2}-2$ & 1.298029942 \\
\hline$z^{6}+z^{5}-z-2$ & 1.081621969 & $z^{6}-z^{4}-2$ & 1.302160040 \\
\hline$z^{5}+z^{4}+z^{3}-2 z-2$ & 1.085235385 & $z^{4}-z^{3}+z-2$ & 1.308571201 \\
\hline$z^{5}+z^{4}+z^{3}-z^{2}-z-2$ & 1.092591837 & $z^{6}-2 z^{2}-2$ & 1.330147493 \\
\hline$z^{6}+z^{5}-z^{4}+z^{2}-z-2$ & 1.097814135 & $z^{5}-z^{3}-2$ & 1.347867896 \\
\hline$z^{6}+z^{4}-z^{2}-2$ & 1.097984258 & $z^{3}-z^{2}+z-2$ & 1.353209964 \\
\hline$z^{5}+z^{4}-z-2$ & 1.102172080 & $z^{6}+z^{5}-z^{3}-2 z^{2}-2 z-2$ & 1.358576643 \\
\hline$z^{4}+2 z^{3}-2 z-2$ & 1.106919340 & $z^{5}-z^{4}-z^{3}+z^{2}+z-2$ & 1.373837398 \\
\hline$z^{6}+z^{3}-2 z^{2}+z-2$ & 1.120923622 & $z^{6}-z^{4}-z-2$ & 1.385332777 \\
\hline$z^{6}-2$ & 1.122462048 & $z^{5}-2 z^{4}+2 z^{3}-2 z^{2}+2 z-2$ & 1.38 \\
\hline$z^{5}+z^{3}-z^{2}-2$ & 1.129131462 & $z^{5}-z^{2}-z-2$ & 1.398813787 \\
\hline$z^{5}+z^{2}-z-2$ & 1.131130966 & $z^{2}-2$ & 1.41 \\
\hline$z^{4}+z^{3}-z-2$ & 1.136528133 & $z^{5}-z^{4}+z^{3}-2 z^{2}+z-2$ & 1.424054221 \\
\hline$z^{5}-2$ & 1.148698355 & $z^{6}-z^{5}-z^{2}+z-2$ & 1.43 \\
\hline$z^{6}+z^{5}-z^{2}-z-2$ & 1.158295133 & $z^{5}+z^{4}-2 z^{2}-3 z-2$ & 1.439011761 \\
\hline$z^{6}-z^{4}+z^{2}-2$ & 1.163275532 & $z^{6}-z^{5}-z^{4}+z^{2}+z-2$ & 1.44 \\
\hline$z^{6}-z^{5}+z^{4}-z^{2}+z-2$ & 1.164546379 & $z^{5}-z^{4}-2$ & 1.45 \\
\hline$z^{5}+z^{4}+z^{3}-z^{2}-2 z-2$ & 1.172622951 & $z^{5}-z^{3}-z-2$ & 1.45 \\
\hline$z^{5}-z^{3}+z^{2}-2$ & 1.175246966 & $z^{6}-2 z^{5}+z^{4}-z^{2}+2 z-2$ & 1.4978 \\
\hline$z^{5}-z^{4}+2 z^{3}-2 z^{2}+z-2$ & 1.176074600 & $z^{6}-z^{3}-2 z^{2}-z-2$ & 1.499508313 \\
\hline$z^{6}-z^{4}+z-2$ & 1.187132205 & $z^{3}-z-2$ & 1.521 \\
\hline$z^{4}-2$ & 1.189207115 & $z^{5}-z^{4}-2 z^{3}+2 z^{2}+z-2$ & 1.537518976 \\
\hline$z^{3}+z^{2}-z-2$ & 1.205569430 & $z^{3}-2 z^{2}+2 z-2$ & 1.543689013 \\
\hline$z^{5}-z^{4}+z^{3}-z^{2}+z-2$ & 1.214862322 & $z^{5}-z^{4}-z^{3}+z^{2}-2$ & 1.564477141 \\
\hline$z^{4}-z^{2}+z-2$ & 1.229001506 & $z^{4}-z^{2}-z-2$ & 1.566383277 \\
\hline$z^{6}-z^{2}-2$ & 1.233442219 & $z^{5}-2 z^{4}+z^{3}-z^{2}+2 z-2$ & 1.581600652 \\
\hline$z^{6}-z^{5}+z^{3}-2 z^{2}+2 z-2$ & 1.237966888 & $z^{5}-z^{4}-z^{2}-2$ & 1.643900159 \\
\hline$z^{6}-2 z^{4}+2 z^{2}-2$ & 1.242452821 & $z^{5}-z^{3}-z^{2}-2 z-2$ & 1.661838867 \\
\hline$z^{6}-z^{5}+z-2$ & 1.248170411 & $z^{3}-z^{2}-2$ & 1.695620770 \\
\hline$z^{3}-2$ & 1.259921050 & $z^{4}-2 z^{3}+2 z-2$ & 1.716672749 \\
\hline$z^{5}-z-2$ & 1.267168305 & $z^{3}-2 z-2$ & 1.769292354 \\
\hline$z^{5}-z^{4}+z-2$ & 1.274707643 & $z-2$ & 2. \\
\hline
\end{tabular}

Corollary 7.1. There exists infinitely many Garsia numbers with minimal polynomial $g(z)$ such that $g(z)$ cannot be written as $g(z)=p(z)+q(z)$, where $p(z)$ and $q(z)$ are products of cyclotomic polynomials.

\section{FinAl COMMENTS}

In this paper we have started the examination of Garsia numbers, and in particular, positive real Garsia numbers. There are many questions raised by this investigation, which we summarize below.

(1) Are all limit points of the form $z^{n}-z^{k}-1$ ?

(2) Are all positive real Garsia numbers approaching these limit points of the form given in equation (11)?

(3) What can be said if $f(z)$ in equation (11) is allowed to be cyclotomic, or allowed to have no real roots? There are examples of such $f(z)$, but nothing is currently known about the structure of such $f(z)$. 
(4) Notice that if $\lambda$ is a limit point of Garsia numbers, then so are the complex values $\sqrt[n]{ \pm \lambda}$ for all $n$. Are there any nonobvious complex limit points of the set of complex limit points?

(5) Can we somehow modify the algorithm in Section 2 to work for any real interval, or any complex region? Currently what we do is find all Garsia numbers of some fixed degree, and then filter the results.

(6) The obvious variation of these polynomials, where norm $(\gamma)= \pm m$ is studied in [8. Which results, if any, have analogous results in this more general setting?

\section{ACKNOWLEDGEMENT}

The authors are grateful for many useful discussions with Boris Solomyak, who had originally proposed this question, and with Chris Smyth.

\section{REFERENCES}

[1] Mohamed Amara, Ensembles fermés de nombres algébriques, Ann. Sci. École Norm. Sup. (3) 83 (1966), 215-270 (1967). MR0237459(38:5741)

[2] David W. Boyd, Pisot and Salem numbers in intervals of the real line, Math. Comp. 32 (1978), no. 144, 1244-1260. MR0491587 (58:10812)

[3] _ Pisot numbers in the neighborhood of a limit point. II, Math. Comp. 43 (1984), no. 168, 593-602. MR758207 (87c:11096b)

[4] no. 1, 17-43. MR804914(87c:11096a)

[5] Horst Brunotte, On Garcia numbers, Acta Math. Acad. Paedagog. Nyházi. (N.S.) 25 (2009), no. 1, 9-16. MR2505180(2010b:11143)

[6] - A class of quadrinomial Garsia numbers, (preprint).

[7] Péter Burcsi and Attila Kovács, Exhaustive search methods for CNS polynomials, Monatsh. Math. 155 (2008), no. 3-4, 421-430. MR2461586 (2009h:11018)

[8] Qirong Deng, The absolute continuity of a family of self-similar measures, Int. J. Nonlinear Sci. 5 (2008), no. 2, 178-183. MR2390971 (2009j:28018)

[9] Paul Erdös, On a family of symmetric Bernoulli convolutions, Amer. J. Math. 61 (1939), 974-976. MR0000311 (1:52a)

[10] A. M. Garsia, Arithmetic properties of Bernoulli convolutions, Trans. Amer. Math. Soc. 102 (1962), 409-432. MR0137961 (25:1409)

[11] Kevin G. Hare, Home page, http://www.math.uwaterloo.ca/ kghare, 2010

[12] Børge Jessen and Aurel Wintner, Distribution functions and the Riemann zeta function, Trans. Amer. Math. Soc. 38 (1935), no. 1, 48-88. MR1501802

[13] Attila Kovács, Generalized binary numbers systems, Ann. Univ. Sci. Budapest. Sect. Comput 20 (2001), 195-206. MR2241084 (2007b:11006)

[14] James McKee and Chris Smyth, Cyclotomic polynomials with interlacing roots, manuscript.

[15] Yuval Peres, Wilhelm Schlag, and Boris Solomyak, Sixty years of Bernoulli convolutions, Fractal geometry and stochastics, II (Greifswald/Koserow, 1998), Progr. Probab., vol. 46, Birkhäuser, Basel, 2000, pp. 39-65. MR1785620 (2001m:42020)

[16] Raphaël Salem, Algebraic numbers and Fourier analysis, D. C. Heath and Co., Boston, Mass., 1963.

[17] I Schur, Über potenzreihen die im inneren des einheitskreises beschrankt sind., J. Reine Angew. Math. 147 (1917), 205-232.

[18] _ Über potenzreihen die im inneren des einheitskreises beschrankt sind., J. Reine Angew. Math. 148 (1918), 128-145.

[19] C. J. Smyth, On the product of the conjugates outside the unit circle of an algebraic integer, Bull. London Math. Soc. 3 (1971), 169-175. MR0289451 (44:6641)

[20] Boris Solomyak, Notes on Bernoulli convolutions, Fractal geometry and applications: a jubilee of Benoît Mandelbrot. Part 1, Proc. Sympos. Pure Math., vol. 72, Amer. Math. Soc., Providence, RI, 2004, pp. 207-230. MR2112107 (2005i:26026) 
Department of Pure Mathematics, University of Waterloo, Waterloo Ontario, CANADA, N2L 3G1

E-mail address: kghare@math.uwaterloo.ca

Department of Pure Mathematics, University of Waterloo, Waterloo Ontario, CANADA, N2L 3G1

E-mail address: mhpanju@rogers.com 